\section{Steroid Sensitive Nephrotic Syndrome and Steroid Toxicity: What to do Next?}

A 10 year old boy presented to our hospital with nephrotic syndrome relapse and signs of steroid toxicity in the form of posterior capsular cataract, affection of linear growth and stage 2 hypertension, in addition to steroid induced adiposity and fungal infection of the scalp resulting in cicatricial alopecia. He was diagnosed as nephrotic syndrome 6 months back. He went into remission with steroid therapy, but was inadvertently taking steroids in high doses for 5 months. When the dose of steroid was tapered, patient had relapse of nephrotic syndrome. As per IAP consensus guidelines for steroid sensitive nephrotic syndrome [1], relapse is to be treated with steroids first. As this patient is suffering from serious steroid toxicity already, is it appropriate to use second line drugs straightway?

JyOTI SHARMA Assistant Professor, Department of Pediatrics, Dr RPGMC Kangra 176001 , (H.P)

\section{REFERENCE}

sharmajyotidr@yahoo.com

1. Consensus Statement of Management of Steroid Sensitive Nephrotic Syndrome. Indian Pediatric Nephrology Group, Indian Academy of Pediatrics. Indian Pediatr. 2008;45:203-14.

\section{RESPONSE TO QUERY}

The present patient received inappropriately prolonged therapy with daily prednisolone resulting in severe steroid toxicity. Following stoppage of corticosteroid therapy, he showed a fast relapse, which is a predictor of frequent relapses and thereby further steroid side effects. The management of this patient therefore comprises of induction and maintenance of remission, and prompt therapy for complications of the nephrotic state. Since the disease is expected to resolve by adulthood in most children with steroid sensitive nephrotic syndrome, limiting adverse effects of medications is an important objective of management.

Oral corticosteroids are still the most effective agents for inducing remission in patients with steroid sensitive disease. The response to therapy with prednisolone is predictable and rapid (8-14 days). Other corticosteroid preparations including IV methylprednisolone and deflazacort are not recommended [1,2]. Similarly, there is no experience with the use of cyclophosphamide, levamisole and mycophenolate mofetil for inducing remission in such children. The other class of agents that might be considered for inducing remission is calcineurin inhibitors (cyclosporine, tacrolimus) [2], which reduce proteinuria by $2-4$ weeks and induce complete remission by $6-8$ weeks. However, the slower response to therapy and associated adverse effects do not justify their use in patients with steroid sensitive nephrotic syndrome. Indications where steroids are avoided for inducing remission, and cyclosporine considered instead, are high blood sugar or steroid psychosis [2].

Once remission is induced, patients with frequent relapses, especially those having steroid toxicity should receive treatment with steroid sparing agents [1-3]. For this purpose, therapy with levamisole, cyclophosphamide, mycophenolate mofetil and calcineurin inhibitors is effective and safe [3]. However, there are few comparative studies on the preferred second-line agent [3], and the choice is determined chiefly by patient and physician preference. Compared to others, treatment with alkylating agents or calcineurin inhibitors offers the best prospect of medium-term steroid free remission [2,3].

The present patient should be treated with prednisolone in the standard dose until remission, and then on alternate days for 4 weeks $[1,2]$. Subsequently, in order to maintain remission, therapy with oral cyclophosphamide $(2-2.5 \mathrm{mg} / \mathrm{kg}$ daily) and alternate day prednisolone $\left(20-30 \mathrm{mg} / \mathrm{m}^{2}\right)$ is recommended. A 12 weeks' course of such treatment is likely to result in sustained remission in a significant proportion of patients. Therapy with calcineurin inhibitors is considered if either steroid therapy is contraindicated (see above) or if frequent relapses recur following cyclophosphamide use. While tacrolimus and cyclosporine show similar efficacy in reducing the frequency of relapses and need for corticosteroids, the latter is preferred in patients with hyperglycemia. Therapy with these agents is, however, initiated after confirming normal renal functions and counseling parents regarding the need for close monitoring.

Additional management includes screening for infectious and other complications. An angiotensin converting enzyme inhibitor is preferred for treating hypertension. The child should consume a balanced diet and be physically active. Parents should be counseled regarding the course of the illness, need for compliance with therapy and adverse effects of medications.

Aditi SinHa, Division of Nephrology, Department of Pediatrics, All India Institute of Medical Sciences, New Delhi 110 029, India. 